\title{
Magnetically Ordered State of Cold Fermions on a Decorated Square Lattice
}

\author{
Kazuto Noda • Akihisa Koga • Norio Kawakami • \\ Thomas Pruschke
}

Received: 15 June 2009 / Accepted: 15 October 2009 / Published online: 29 October 2009

(C) Springer Science+Business Media, LLC 2009

\begin{abstract}
We study two-component ultracold fermions with repulsive interactions, which are loaded into a decorated square lattice. By combining the real-space dynamical mean-field theory with the numerical renormalization group method, we discuss magnetic properties in the system. It is clarified how the ferromagnetically ordered ground state, which is stabilized by a flat band mechanism, is adiabatically connected to the ferrimagnetically ordered state expected in the strong coupling limit.
\end{abstract}

Keywords Optical lattice $\cdot$ Fermion $\cdot$ Ferromagnetism

\section{Introduction}

Recently, optical lattice systems have attracted much interest [1]. One of the remarkable examples is a fermionic optical lattice system, which is formed by loading fermionic atoms in a periodic potential [2]. Owing to its high controllability in the interaction strength, the number of particles and other parameters, many remarkable phenomena have been observed such as superfluidity [3], Mott transition [4-6], etc. Current experiments in optical lattices are done at temperatures higher than typical magnetic transition temperature, and therefore a magnetic order has not been observed so far. Nevertheless, the possible realization of magnetically ordered states for cold atoms in optical traps is a very hot topic [1]. Theoretically it has been predicted

K. Noda $(\bowtie) \cdot$ N. Kawakami

Department of Physics, Graduate School of Science, Kyoto University, Kyoto, Japan

e-mail:noda@scphys.kyoto-u.ac.jp

A. Koga

Department of Physics, Tokyo Institute of Technology, Tokyo, Japan

T. Pruschke

Institut für Theoretische Physik, Universität Göttingen, Göttingen, Germany 
Fig. 1 (Color online)

Decorated square lattice.

Dashed line shows the unit cell.

Sketch of the RDMFT

procedure, in detail see text
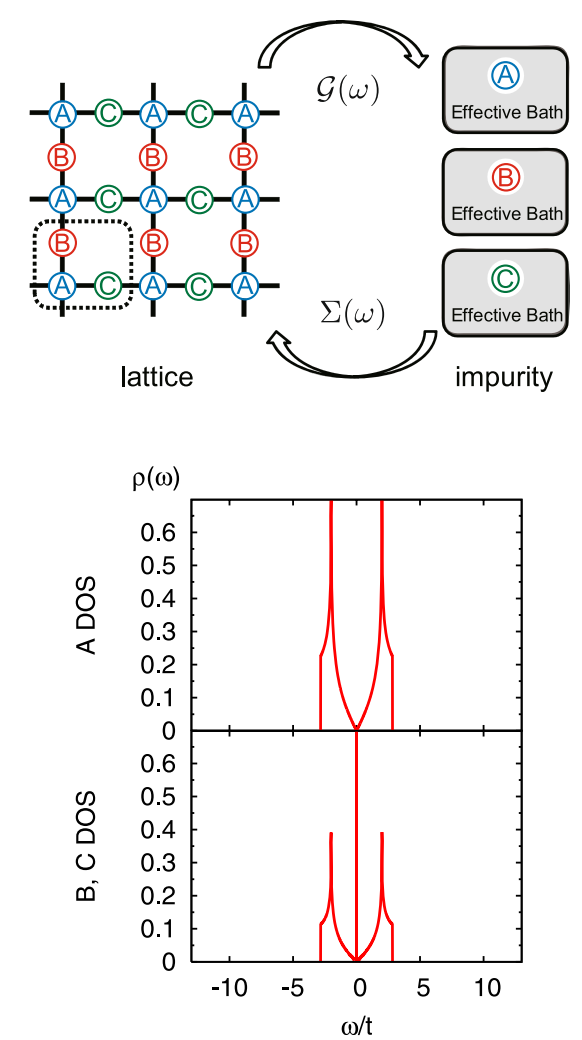

Fig. 2 Local density of states (LDOS) for noninteracting particles. Up panel is LDOS of $A$ site, and down panel is that of $B, C$ site

that magnetic states should be realized in some fermionic optical lattice systems such as a two-dimensional (2D) square lattice with confining potential [7] and a honeycomb lattice with $p$-orbitals [8]. Note that the transition temperature in the 2D model we treat here (see below) is of the same order in magnitude as expected for the above 2D systems. We therefore believe that present study will add to the motivation to intensively study magnetism in cold atom systems.

We here investigate how the magnetic state emerges for an optical lattice with specific geometry that has a tendency to stabilize the ferromagnetism. The model system we consider for this purpose is a decorated square lattice shown in Fig. 1, which may be expected to be realized experimentally in the future. The lattice is composed of three kinds of sites, $\mathrm{A}, \mathrm{B}$ and $\mathrm{C}$, whose characteristic structure gives rise to a flat-band structure in the local density of states (LDOS): a delta-functionlike peak (flat band) appears in the LDOS for B and C sites (see Fig. 2). In the halffilled case, an infinitesimal interaction lifts the degenerate electron states at the Fermi level, which drives the system to the ferromagnetically ordered state, as guaranteed by Lieb's theorem [9]. However, it is still nontrivial and interesting to elucidate how the magnetically ordered state changes its character when the interaction strength is varied. To address this problem, we study magnetic properties in the fermionic optical lattice system on the decorated square lattice. We briefly comment on research activities concerning ferromagnetism in condensed matter physics. The studies based 
on the Hubbard model have been continued since the 1930's. In recent two decades, remarkable progress has been made about flatband ferromagnetism [10-12] and itinerant ferromagnetism in the Hubbard model $[13,14]$. We also note that an attempt to realize flatband ferromagnetism on a decorated square lattice was indeed proposed for quantum dot systems [15].

\section{Model and Hamiltonian}

We consider two-component ultracold fermions on the decorated square lattice, which is described by the following Hubbard Hamiltonian,

$$
H=-\sum_{\langle i, j\rangle, \sigma} t_{i j} c_{i, \sigma}^{\dagger} c_{j \sigma}+U \sum_{i} n_{i \uparrow} n_{i \downarrow}
$$

where $c_{i \sigma}^{\dagger}\left(c_{j \sigma}\right)$ creates (annihilates) a fermion at the $i$ th site with spin $\sigma$, and $n_{i \sigma}=$ $c_{i \sigma}^{\dagger} c_{i \sigma} . t_{i j}$ is the nearest-neighbor hopping and $U$ is a repulsive interaction.

To discuss ground-state properties of the system, we use the real-space dynamical mean-field theory (R-DMFT) [16-25], which has successfully been applied to some correlated systems such as an interface between the band insulator and the Mott insulator [21, 22], repulsive [7, 23] or attractive [24, 25] fermionic atoms with confining potential. In the R-DMFT, the lattice model is mapped onto an effective impurity model, where local correlations are taken into account precisely. In our decorated square lattice model, we need to introduce three kinds of effective impurity models. The Green function of the effective bath $\mathcal{G}_{\alpha \sigma}$, is determined by the Dyson equation,

$$
\hat{\mathcal{G}}_{\alpha \sigma}^{-1}(\omega)=\left[\sum_{\mathbf{k}} \frac{1}{\omega+\mu-\hat{t}(\mathbf{k})-\hat{\Sigma}_{\sigma}(\omega)}\right]^{-1}+\hat{\Sigma}_{\alpha \sigma}(\omega)
$$

where $\mu$ is the chemical potential, $\mathbf{k}$ is the wave vector in the reciprocal lattice space, and $\hat{\Sigma}_{\alpha}$ is the self-energy for the $\alpha$ th sublattices. Here, $\hat{\mathcal{G}}, \hat{\Sigma}, \hat{t}$ are represented by $3 \times 3$ matrices.

To solve the effective impurity models, we employ the numerical renormalization group (NRG) [26-29], where the effective bath is discretized on a logarithmic mesh, enabling us to treat low-energy properties precisely. NRG is especially powerful to study our system, since we should treat correlation effects including a singular behavior due to a flat-band in DOS near the Fermi level. We can therefore access the small energy scales in the system quantitatively. To ensure that the sum rules for dynamical quantities are fulfilled, we use the complete basis set algorithm proposed recently $[30,31]$. In this study, we calculate the particle density $n_{\sigma}$ and the magnetization $m_{\alpha}=\frac{1}{2}\left(n_{\alpha \uparrow}-n_{\alpha \downarrow}\right)$ to discuss the effects of the interaction on the magnetically ordered ground state. We note here that our calculation is done at $T=0$.

\section{Results}

To discuss how the ferromagnetically ordered state is affected by repulsive interactions, we show the magnetization for each sublattice in Fig. 3. Upon introducing the 
Fig. 3 (Color online) Squares (triangles) represent spontaneous magnetizations for A (B and C) sublattices. Crosses represent the total magnetizations per unit cell

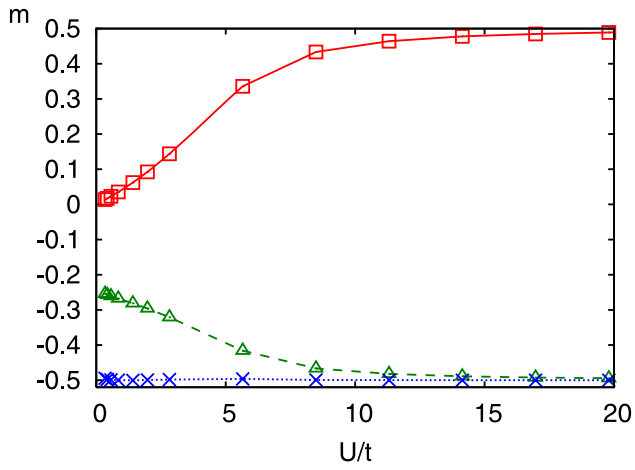

interaction $U$, the magnetization for the A sublattice increases gradually from zero, while the magnetizations for the $\mathrm{B}$ and $\mathrm{C}$ sublattices appear discontinuously from zero to $m=-0.25$. This singular behavior is caused by the flat band structure at the Fermi level in the LDOS (see Fig. 2). The resulting ferromagnetism is often called the flat-band ferromagnetism [9]. As the interaction further increases, the magnetization for each sublattice increases gradually and approaches the saturated values in the strong coupling regime, where the ferrimagnetically ordered ground state is realized with staggered magnetizations $\left(m_{A} \sim 0.5\right.$ and $\left.m_{B, C} \sim-0.5\right)$. Therefore, there is a smooth crossover from the flat-band ferromagnetism to the Heisenberg-type ferrimagnetism when the strength of $U$ is varied. We note that the total magnetization of the unit cell is always preserved during the change of the interaction strength, which is consistent with Lieb's theorem.

What happens in the weak- and strong coupling regime can be more clearly seen in the LDOS for each sublattice. We first recall that at $U=0$ (Fig. 2), the LDOS has the flat band in the B, C sublattices at the Fermi level, but not in the A sublattice. When an infinitesimal interaction is introduced, the splitting of the flat band at the Fermi level (Fig. 4(a) and (b)) leads to the sudden increase of magnetizations for the B and $\mathrm{C}$ sublattices. The weight of the flat band is half of the total weight in DOS, resulting in the total magnetization $m_{\text {tot }}=-0.5$ per unit cell in accordance with the computed results in Fig. 3. Therefore, we can see that the ferromagnetism in the weak coupling regime is dominated by the electrons around the Fermi level in the B and C sublattices. On the other hand, in the strong coupling regime, where the magnetization for each sublattice is almost saturated, all the electrons in the entire energy region contribute to the formation of the Heisenberg-type ferrimagnetic order (Fig. 4). Note that the crossover occurs around $U / W \sim 1$, where $W(=4 \sqrt{2})$ means the bandwidth. Let us discuss the crossover from a ferromagnetic to ferrimagnetic state under adiabatic evolution in some more detail. The flatband ferromagnetism is caused by a flatband at the Fermi level in the weak coupling region, where only the $\mathrm{B}$ and $\mathrm{C}$ sublattices are polarized ferromagnetically with a vanishing magnetization of the A sublattice. As $U$ increases, a magnetic moment is induced gradually on the A sublattice in a direction opposite to the ferromagnetic order. Note, however, that the total magnetization is always conserved (cf. also Fig. 3). Therefore, the flatband ferromagnetism can actually be considered as an extreme limit of the ferrimagnetism, although the physics stabilizing a magnetic order have different origins for these two states. A similar example 

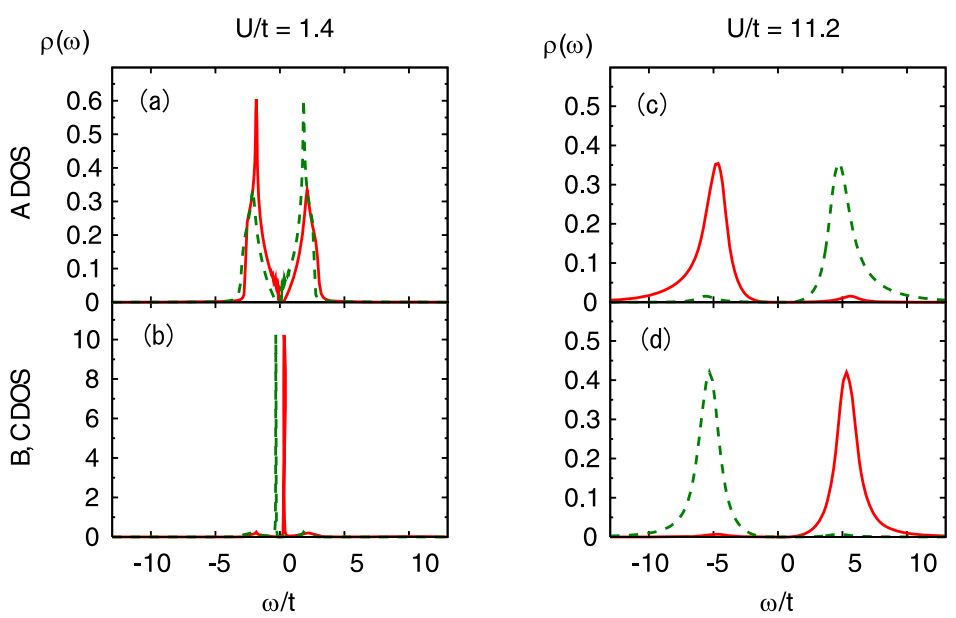

Fig. 4 (Color online) Solid (dashed) lines represent the LDOS for up (down) spin in the system with $U / t=1.4$ (left) and 11.2 (right)

of such a crossover can be found in condensed matter physics, where the DMFT study in the cubic lattice revealed a crossover from a spin density wave in the weak coupling to a Heisenberg antiferromagnet in the strong coupling limit [32].

\section{Summary}

In summary, we have investigated the two-component fermionic repulsive Hubbard model in a decorated square lattice. Using R-DMFT combined with NRG, we have revealed how the system exhibits a crossover from the flat-band ferromagnetism in the weak coupling regime to the Heisenberg ferrimagnetism in the strong coupling regime. In order to observe the above magnetic state experimentally in an optical lattice, it is crucial to examine how stable it is at finite temperatures. It is known that in the pure $2 \mathrm{D}$ case addressed here, a phase transition should not occur at finite temperatures. However, if we consider quasi-2D systems, we can expect a finite-temperature phase transition. It can be shown that the corresponding transition temperature should become highest in the crossover regime. This encourages experimental investigations on a quasi-2D optical lattice system in the crossover regime. The detailed analysis of quasi-2D cases is now under investigation.

In this paper, we have neglected, for simplicity, the effects of the energy-level splitting, the hopping imbalance, etc., which should be also important to discuss the stability of the magnetically ordered state in the optical lattice. These effects will be addressed elsewhere.

Acknowledgements We thank Y. Takahashi for fruitful discussions and valuable comments on realization of the decorated square lattice system in cold atom systems. This work was partly supported by the Grant-in-Aid for Scientific Research [20029013, 21540359 (N.K.) and 20740194 (A.K.)] and the Grant-inAid for the Global COE Programs "Next Generation of Physics, Spun from Universality and Emergence" and "Nanoscience and Quantum Physics" from the Ministry of Education, Culture, Sports, Science and Technology (MEXT) of Japan. 


\section{References}

1. I. Bloch, J. Dalibard, W. Zwerger, Rev. Mod. Phys. 80, 885 (2008)

2. I. Bloch, Nat. Phys. 1, 23 (2005)

3. J.K. Chin, D.E. Miller, Y. Liu, C. Stan, W. Setiawan, C. Sanner, K. Xu, W. Ketterle, Nature 443, 961 (2006)

4. R. Jördens, N. Strohmaier, K. Günter, H. Moritz, T. Esslinger, Nature 455, 204 (2008)

5. U. Schneider, L. Hackermüller, S. Will, Th. Best, I. Bloch, T.A. Costi, R.W. Helmes, D. Rasch, A. Rosch, Science 322, 1520 (2008)

6. S. Trotzky, P. Cheinet, S. Fölling, M. Feld, U. Schnorrberger, A.M. Rey, A. Polkovnikov, E.A. Demler, M.D. Lukin, I. Bloch, Science 319, 295 (2008)

7. M. Snoek, I. Titvinidze, C. Toke, K. Byczuk, W. Hofstetter, New J. Phys. 10, 093008 (2008)

8. S. Zhang, H. Hung, C. Wu, arXiv:0805.3031

9. E.H. Lieb, Phys. Rev. Lett. 62, 1201 (1989)

10. A. Mielke, J. Phys. A 24, L73 (1991)

11. A. Mielke, J. Phys. A 24, 3311 (1991)

12. H. Tasaki, Phys. Rev. Lett. 69, 1608 (1992)

13. M. Ulmke, Eur. Phys. J. B 1, 301 (1997)

14. J. Wahle, N. Blümer, J. Schlipf, K. Held, D. Vollhardt, Phys. Rev. B 58, 12749 (1998)

15. H. Tamura et al., Phys. Rev. B 65, 085324 (2002)

16. W. Metzner, D. Vollhardt, Phys. Rev. Lett. 62, 324 (1989)

17. E. Müller-Hartmann, Z. Phys. B 74, 507 (1989)

18. Th. Pruschke, M. Jarrell, J.K. Freericks, Adv. Phys. 44, 187 (1995)

19. A. Georges, G. Kotliar, W. Krauth, M.J. Rozenberg, Rev. Mod. Phys. 68, 13 (1996)

20. G. Kotliar, D. Vollhardt, Phys. Today 57, 53 (2004)

21. S. Okamoto, A.J. Millis, Phys. Rev. B 70, 241104 (2004)

22. S. Okamoto, A.J. Millis, Nature (Lond.) 428, 630 (2004)

23. R.W. Helmes, T.A. Costi, A. Rosch, Phys. Rev. Lett. 100, 056403 (2008)

24. A. Koga, T. Higashiyama, K. Inaba, S. Suga, N. Kawakami, J. Phys. Soc. Jpn. 77, 073602 (2008)

25. A. Koga, T. Higashiyama, K. Inaba, S. Suga, N. Kawakami, Phys. Rev. A 79, 013607 (2009)

26. K.G. Wilson, Rev. Mod. Phys. 47, 773 (1975)

27. H.R. Krishna-murthy, J.W. Wilkins, K.G. Wilson, Phys. Rev. B 21, 1003 (1980)

28. H.R. Krishna-murthy, J.W. Wilkins, K.G. Wilson, Phys. Rev. B 21, 1044 (1980)

29. R. Bulla, T.A. Costi, Th. Pruschke, Rev. Mod. Phys. 80, 395 (2008)

30. F.B. Anders, A. Schiller, Phys. Rev. Lett. 95, 196801 (2005)

31. R. Peters, Th. Pruschke, F.B. Anders, Phys. Rev. B 74, 245114 (2006)

32. Th. Pruschke, Prog. Theor. Phys. Suppl. 160, 274 (2005) 